\title{
LETTER
}

\section{Innovative single-shot 2D pulse front tilt diagnostic}

\author{
M. Galimberti ${ }^{1}$, F. G. Bisesto ${ }^{2}$, and M. Galletti ${ }^{2}$ \\ ${ }^{1}$ Central Laser Facility, Science and Technology Facilities Council, Rutherford Appleton Laboratory, Didcot, UK \\ ${ }^{2}$ INFN Laboratori Nazionali di Frascati, Frascati, Italy \\ (Received 19 November 2020; revised 21 December 2020; accepted 24 December 2020)
}

\begin{abstract}
The presence of pulse front tilt (PFT), caused by angular dispersion (AD) in femtosecond laser pulses, could degrade the performance of the laser system and/or impact the experimental yields. We present a single-shot diagnostic capable of measuring the $\mathrm{AD}$ in the $x-y$ plane by adopting an intensity mask. It can be applied to stretched pulses, making it ideal for diagnosing the $\mathrm{AD}$ along the amplification chain of a high-power laser system, and to ultrashort pulses exiting from an optical compressor. In this way, it can help in properly characterizing a laser pulse before it is delivered to the target area. In this Letter, we present experimental evidence of AD retrieval for different compression configurations, supported by theoretical analysis.
\end{abstract}

Keywords: femtosecond pulses; high-power laser; laser diagnostics; pulse front tilt

The development of the ultrafast laser system opened up a large number of new research fields. New optical techniques have been developed to reduce the pulse duration to a few femtoseconds and/or to increase the peak power to the multi-petawatt range. The generation of ultrashort pulses implies a large bandwidth, increasing complexity of the beam propagation, and diagnostics to fully characterize the final pulse in the spatiotemporal domain. One of the spatiotemporal couplings $(\mathrm{STCs})^{[1]}$ playing a key role in a wide range of scientific areas, e.g., laser development, lasermatter interaction, is the angular dispersion (AD) of the beam. The presence of this chromatic aberration distorts the laser pulse, causing pulse front tilt $(\mathrm{PFT})^{[2]}$. In turn, when the beam is focused, the actual pulse length in the focal spot is different from the pulse length in the near field, where it is usually measured.

The presence of PFT in the near field has been proved to have an effect on electron acceleration experiments ${ }^{[3]}$, so its control and measurement are important for the development of compact laser-driven plasma accelerators and, more generally, to guarantee the best performance of high-power laser facilities.

Correspondence to: M. Galimberti, Central Laser Facility, Science and Technology Facilities Council, Rutherford Appleton Laboratory, Harwell Science and Innovation Campus, Didcot, OX11 0QX, UK. Email: marco.galimberti@stfc.ac.uk
In high-power ultrashort laser systems, slight misalignment of the compressor and/or the stretcher can generate $\mathrm{AD}^{[4]}$. Different diagnostics ${ }^{[5]}$ have been proposed to characterize these aberrations, based on interferometric ${ }^{[6,7]}$, spectral-temporal $^{[8]}$, autocorrelation ${ }^{[9]}$, chromatic $^{[10]}$ or wave-front distortion ${ }^{[11]}$ techniques. Unfortunately, these diagnostics involve complex setup ${ }^{[12-14]}$, originating from complex alignment procedures, and most of them are selfreferenced ${ }^{[15]}$, so they need a good-quality beam, both in the spatial and the temporal domains. Moreover, they involve complex retrieval routines, sometimes leading to ambiguities in the detection of $\mathrm{STCS}^{[7]}$ or providing only qualitative measurements.

In this Letter, we propose a simple innovative singleshot method, based on an intensity mask. It is suitable for characterizing not only short pulses, as required for many available diagnostics, but also stretched pulses. The proposed instrument addresses most of the main issues of existing STC diagnostics: it is a simple retrieval routine able to retrieve different STCs without ambiguities; the simple experimental setup involves simple alignment procedures and the instrument is not a self-referenced method so it does not require a good-quality beam in the spatiotemporal domain. Above all, the main advantage of this new diagnostic is the simplicity of the setup and the ability to show, directly from the acquired image, the presence of PFT prior to the analysis in both directions. The theoretical background and the diagnostic 
performances are first presented together with simulations, in detail. Second, experimental results, supported by the theoretical analysis of the beam propagation, are reported, confirming the feasibility of our tool.

The basic idea of the proposed instrument is to use a diffraction element to disperse the spectral components angularly. If a transmission grating is used as the diffraction element in the horizontal direction, by focusing the diffraction order and analyzing the diffracted spot, the spectral components are diffracted at different horizontal positions while the vertical position encodes information about the AD. By using a 2D diffraction element and analyzing the diffraction orders in both directions, the AD in both directions can be retrieved. To study in detail the theory behind the instrument, let us consider a low aberrated laser beam that can be described by an electric field $E(r, \lambda)$ and an intensity profile $I(r, \lambda)$ independent of the wavelength $\lambda$, i.e.,

$$
I(r, \lambda) \propto|E(r, \lambda)|^{2} \propto I(r)
$$

where $r=(x, y)$ determines the spatial coordinates in the $x$ $y$ plane. In the proposed study, the beam polarization is not relevant; thus the electric field is considered to be a scalar quantity.

In the paraxial approximation, adopting an achromatic lens of focal length $f$, the far-field spectral intensity $S(r, \lambda)$ is given by

$$
S(r, \lambda)=\frac{1}{\lambda^{2} f^{2}}\left|\int E(\rho, \lambda) \exp \left(-i \frac{2 \pi}{\lambda f} r \cdot \rho\right) \mathrm{d} \rho\right|^{2},
$$

where $r$ and $\rho$ are, respectively, the spatial coordinates in the far field and in the near field. Here $E(\rho, \lambda)$ is described by

$$
E(\rho, \lambda)=\mathcal{M}(\rho) E_{0}(\rho, \lambda) \exp \left[+i \frac{2 \pi}{\lambda} \bar{\alpha}(\lambda) \cdot \rho\right]
$$

where $\bar{\alpha}(\lambda)$ is the pointing direction for each wavelength $\lambda$ and $\mathcal{M}(\rho)=\sum_{i} \chi(\rho-d i)$ is the periodic amplitude mask where $\chi$ is the function describing the mask profile and $d$ its periodicity. From Equations (1) and (2), the far-field spectral intensity $S(r, \lambda)$ becomes

$$
S(r, \lambda)=\sum_{n} \mathcal{G}_{n} S_{0}\left[r-f \bar{\alpha}(\lambda)-\frac{\lambda f}{d} n, \lambda\right]
$$

where $n$ is a vector of integers representing the order of diffraction generated by the mask, $\mathcal{G}_{n}$ is the amplitude factor for each order and $S_{0}(r, \lambda)$ is the spectral intensity profile of the unperturbed far field. Apart from the central spot $(n=$ $(0,0))$, which represents the unperturbed beam, the spots are spatially dispersed by the grating effect of the intensity mask.
Let us now consider a Gaussian beam described by

$$
S_{0}(r, \lambda)=I_{0} \exp \left[-\frac{1}{2} \frac{|r|^{2}}{\sigma^{2}}-\frac{1}{2}\left(\frac{\lambda-\lambda_{0}}{\Delta}\right)^{2}\right] .
$$

where $\sigma$ is related to the spot size, $\lambda_{0}$ is the laser central wavelength and $\Delta$ is related to the pulse bandwidth. Assuming a linear and relatively small $\mathrm{AD}$ (respectively, $\bar{\alpha}(\lambda)=$ $\alpha\left(\lambda-\lambda_{0}\right)$ and $\left.|\alpha| \ll 1 / d\right)$ and just the first diffraction order $(|n|=1)$, the intensity profile $I_{x}^{ \pm}$of the diffraction order $n=( \pm 1,0)$ is

$I_{x}^{ \pm}(x, y) \propto \exp \left\{-\frac{1}{2}\left\{\left(\frac{x-x_{ \pm}}{\Omega_{x}^{ \pm}}\right)^{2}+\left[\frac{y-A_{x}^{ \pm}\left(x-x_{ \pm}\right)}{\sigma}\right]^{2}\right\}\right\}$,

where

$$
\begin{gathered}
\Omega_{x}^{ \pm}=\frac{f \Delta}{d} \sqrt{\Gamma \pm 2 d \alpha_{x}}, \quad A_{x}^{ \pm}= \pm\left(\Gamma \pm 2 d \alpha_{x}\right)^{-1} d \alpha_{y}, \\
x_{ \pm}= \pm \frac{f \lambda_{0}}{d}, \quad \Gamma=1+\left(\frac{d \sigma}{\Delta f}\right)^{2} .
\end{gathered}
$$

Equations (5) and (6) are also valid for the $y$-coordinate by just replacing $x$ with $y$. The $\mathrm{AD} \alpha$ can be obtained by solving the linear system

$$
\begin{aligned}
& \alpha_{x}-\left(A_{y}^{+}+A_{y}^{-}\right) \alpha_{y}=\frac{\Gamma}{2 d}\left(A_{y}^{+}-A_{y}^{-}\right), \\
& \alpha_{y}-\left(A_{x}^{+}+A_{x}^{-}\right) \alpha_{x}=\frac{\Gamma}{2 d}\left(A_{x}^{+}-A_{x}^{-}\right),
\end{aligned}
$$

while the scaling factor $\Gamma$ can be obtained from $\Omega_{x}^{ \pm}$and $\sigma$ by

$$
\Gamma=1+\left[1-\frac{\left(\Omega_{x}^{+}\right)^{2}+\left(\Omega_{x}^{-}\right)^{2}}{2 \sigma^{2}}\right]^{-1} .
$$

Starting from the image and considering only the diffraction order $n=(+1,0)$, the parameters $A_{x}^{+}, \Omega_{x}^{+}$and $\sigma$ can be retrieved by analyzing the diffracted spot. With a fixed position on the $x$-axis, the barycenter $y_{\mathrm{B}}$ and the root mean square deviation (RMSD) $\sigma_{\mathrm{B}}$ are calculated along the $y$-axis. The average of $\sigma_{\mathrm{B}}$ provides the estimation for $\sigma$, while the RMSD value along the $x$-axis, after integrating along the $y$ axis, gives an estimation of $\Omega_{x}^{+}$. A linear regression of $y_{\mathrm{B}}$ retrieves the slope $A_{x}^{+}$(Figure 1 ).

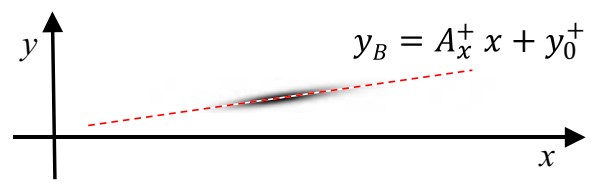

Figure 1. Simulated image of the first diffracted spot $(n=(+1,0))$ in $x$ with the linear regression to retrieve the slope $A_{x}^{+}$. 
The focal length of the lens has a direct implication for the resolution of the instrument: a longer focal length creates a larger deviation, encoding the information on a larger number of pixels. However, it also requires a larger camera sensor to be able to collect the first orders of diffraction. So, with the parameters of the camera (number of pixels $\mathcal{N}$ and pixel size $\left.\sigma_{\mathrm{pxl}}\right)$ and the period of the mask $(d)$ fixed, the optimal focal length $f_{\text {opt }}$ should be chosen to maximize the separation of the first orders, avoiding clipping at the edge of the bandwidth, i.e.,

$$
f_{\mathrm{opt}} \approx \frac{\mathcal{N} \sigma_{\mathrm{pxl}} d}{2\left(\lambda_{0}+\Delta\right)}
$$

From the AD values $\alpha$, it is easy to obtain the PFT and PFT angles $\zeta$ in the near field:

$$
\mathrm{PFT}=\lambda_{0} \frac{\alpha}{c}, \tan \zeta=\lambda_{0} \alpha
$$

where $c$ is the speed of light in a vacuum.

To validate the instrument, a software program was developed to simulate the focusing of a broad-bandwidth laser beam. The program takes an arbitrary shape of the input beam in the spectrum-space domain and simulates the focusing described by Equation (1). For each wavelength, a numerical Fourier transform is performed, obtaining the simulated focal spot images. Each image is then re-scaled to the same resolution and integration over the wavelength range is performed to obtain the final focal spot image. The program is implemented using the ArrayFire library ${ }^{[16]}$ and the CUDA library and it was run on a PC with an NVIDIA M6000 graphics card. All the simulations were performed using a $2048 \times 2048$ point array with a pixel size of $5.3 \mu \mathrm{m}$ and 64 samplings in the spectral domain. The camera had a resolution of $1024 \times 1024$ pixels, a squared mask of $100 \mu \mathrm{m}$ with a periodicity of $d=156 \mu \mathrm{m}$ (commercially available) and a lens with a focal length of $f=300 \mathrm{~mm}$. These parameters were chosen to match both the camera and the amplitude mask used in the experimental validation, presented below. To generate more realistic images, some white noise was added and digitization to 8-bit dynamics was considered. Figure 2 shows a simulated image for a Gaussian beam, similar to that in the experimental case, of $7.65 \mathrm{~mm}$ full width at half maximum (FWHM), with a Gaussian spectrum having a central wavelength $\lambda_{0}=800 \mathrm{~nm}, 25 \mathrm{~nm}$ FWHM bandwidth and $\mathrm{AD}$ of $\alpha=(11.5,0) \mu \mathrm{rad} / \mathrm{nm}$. The intensity level was chosen to maximize the dynamics on the first-order diffracted spots, clearly visible in the image.

Starting from the image (simulated or experimental) of the focal plane (Figure 2), a background subtraction algorithm and numerical filtering were performed. The resulting image was centred and each diffracted spot was analyzed to obtain the parameters $A_{x}^{ \pm}, A_{y}^{ \pm}, \Omega_{x}^{ \pm}, \Omega_{y}^{ \pm}$and $\sigma$, as defined in Equation (6). Using Equation (8) to estimate $\Gamma$ and solving

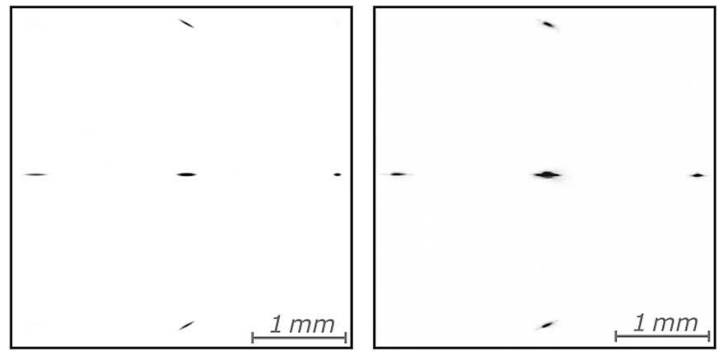

Figure 2. Camera image comparison for a $7.65 \mathrm{~mm}$ FWHM Gaussian beam with $25 \mathrm{~nm}$ FWHM Gaussian spectrum around $800 \mathrm{~nm}$ and AD of $\alpha=(11.5,0) \mu \mathrm{rad} / \mathrm{nm}$ : (left) simulated image; (right) experimental image.

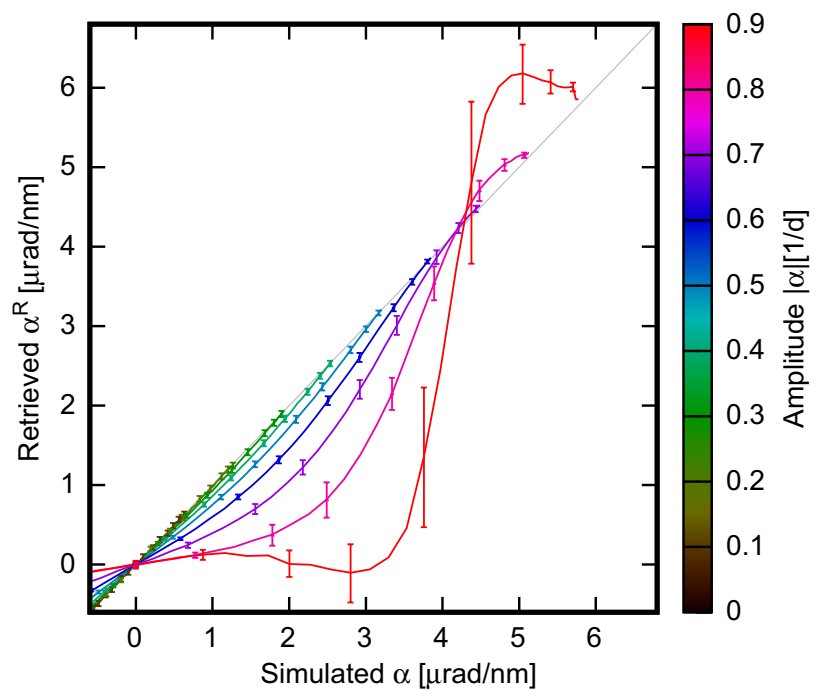

Figure 3. Retrieved versus simulated $\mathrm{AD}$ for a $50 \mathrm{~nm}$ FWHM Gaussian spectrum and $5 \mathrm{~mm}$ FWHM Gaussian beam for a different AD modulus.

the system of Equation (7), we obtained the retrieved $\mathrm{AD}$ values $\alpha^{\mathrm{R}}$. The estimation of the error on the retrieved AD was performed by taking the noise level of the image and using the error propagation theory.

A set of images, having different amplitude of AD values as $\alpha=(t / d \cos \theta, t / d \sin \theta)$ where $t / d$ is the $\mathrm{AD}$ modulus and $\theta$ the direction, was used to numerically test the instrument. The simulated beam was a Gaussian beam of $5 \mathrm{~mm}$ FWHM and had a Gaussian spectrum with a central wavelength of $\lambda_{0}=800 \mathrm{~nm}$ and a bandwidth of $50 \mathrm{~nm}$ FWHM. The plot of the retrieved $\alpha_{x}^{\mathrm{R}}$ and $\alpha_{y}^{\mathrm{R}}$ versus the simulated $\alpha_{x}$ and $\alpha_{y}$ $\mathrm{AD}$ values is shown in Figure 3. The plot shows a clear agreement between the simulated and the retrieved values for a small value of $|\alpha|$, where the condition

$$
|\alpha| \ll 1 / d \simeq 6.41 \mu \mathrm{rad} / \mathrm{nm}
$$

is fulfilled. In more detail, the induced error is $0.018 \mu \mathrm{rad} / \mathrm{nm}$ for $|\alpha|=0.2 / d$ and $0.041 \mu \mathrm{rad} / \mathrm{nm}$ for $|\alpha|=0.3 / d$, compared with the estimated measurement error of $0.033 \mu \mathrm{rad} / \mathrm{nm}$ for both cases. At larger AD amplitude, there is poor agreement between the retrieved and the simulated 
Table 1. Influence of the beam and spectral shape.

\begin{tabular}{lllc}
\hline Beam shape & Spectral shape & $\eta$ & $\sigma_{\text {err }}(\mu \mathrm{rad} / \mathrm{nm})$ \\
\hline Gaussian & Gaussian & 1 & 0.018 \\
Gaussian & Flat-top & 1 & 0.020 \\
Square flat-top & Gaussian & 0.86 & 0.020 \\
Square flat-top & Flat-top & 0.85 & 0.022 \\
\hline
\end{tabular}

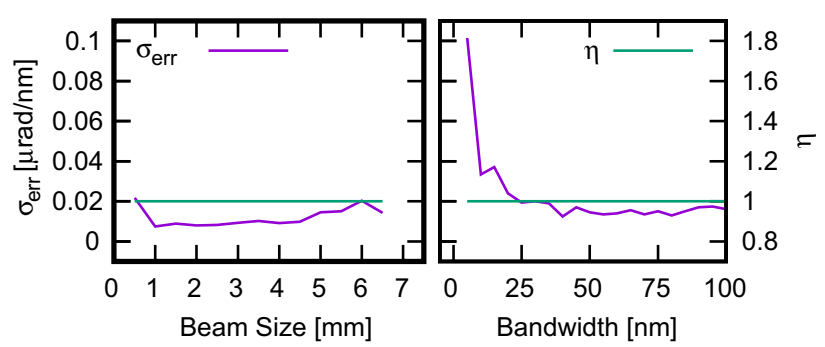

Figure 4. Influence on calibration $\eta$ and retrieved error $\sigma_{\mathrm{err}}$ of various beam parameters: (left) beam size FWHM; (right) spectral bandwidth FWHM.

$\mathrm{AD}$, except when the $\mathrm{AD}$ is in the diffraction direction of the intensity mask, i.e., $\theta=0, \theta=\pi$ or $\theta= \pm \pi$, where the induced error is of the order of $0.033 \mu \mathrm{rad} / \mathrm{nm}$. In this case, the maximum measurable amplitude of the $\mathrm{AD}$ is limited only by the dimension of the camera, which can cause clipping on the diffracted spots, and superimposition between the first and the second diffraction orders. To fully test the instrument, different beam parameters were simulated using the $\mathrm{AD}$ of $|\alpha|=\frac{0.2}{d} \simeq 1.28 \mu \mathrm{rad} / \mathrm{nm}$ and different $\theta$, keeping the same parameters as in the previous simulations. The retrieved $\mathrm{AD} \alpha^{\mathrm{R}}$ was analyzed, performing a linear fit, namely

$$
\alpha_{x, y}^{\mathrm{R}}=\eta \alpha_{x, y},
$$

extracting the systematic calibration error $\eta$ and standard deviation of the residual $\sigma_{\text {err }}$. Varying the shape of the spectrum from Gaussian to flat-top, no substantial influence on the retrieved AD is noticed, as shown in Table 1. On the other hand, the spatial shaping of the beam in the near field (from Gaussian to square flat-top) caused the retrieved $\mathrm{AD}$ to be underestimated by $\sim 15 \%$. The near-field beam size has a marginal influence, while the decrease of the spectral bandwidth below $\approx 25 \mathrm{~nm}$ affects the retrieval, where the retrieved error increases with the reduction of the bandwidth (Figure 4). The proposed technique, in its current status, cannot manage the overlapping of the second order with the first order of diffraction. A possible workaround is to insert a known $\mathrm{AD}$ at $45^{\circ}$ from the diffraction direction by a prism, to separate the dispersion orders. The analysis routine could be easily modified to take into account the additional AD.

The presence of astigmatism is well tolerated if small $\left(<0.2 \lambda_{0}\right.$ of peak-to-valley aberration), while for higher values it largely degrades the retrieved error. Also, the

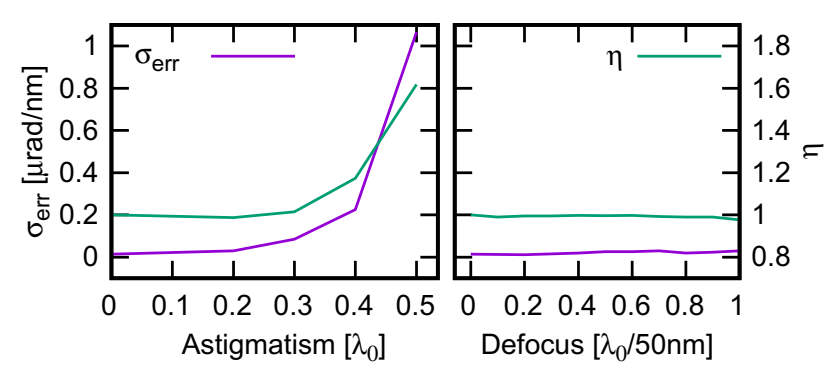

Figure 5. Influence on calibration $\eta$ and retrieved error $\sigma_{\text {err }}$ of various beam parameters: (left) astigmatism; (right) chromatic defocus.

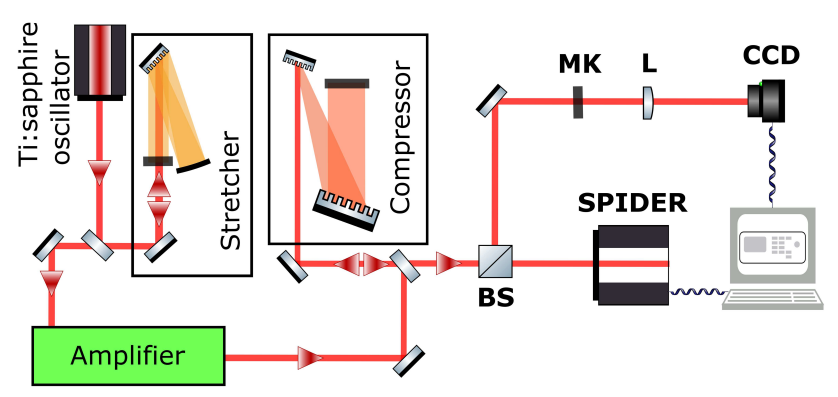

Figure 6. Schematic of the experimental setup implemented in the front end of the FLAME laser system. BS, beam splitter; CCD, charge-coupled device camera; L, achromatic doublet; MK, diffractive mask.

calibration error $\eta$ became significantly different from 1 for aberration greater than $0.3 \lambda_{0}$ (Figure 5 (left)). It should be emphasized that, for each aberration value, the angular direction of the astigmatism was chosen randomly. Finally, the chromatic defocusing is well tolerated by the instrument, with a modest increase of the retrieved error with increase of the aberration (Figure 5 (right)). The instrument was validated experimentally with the FLAME laser ${ }^{[17]}$, exploiting the auxiliary beamline usually used as probe beam ${ }^{[18]}$. Figure 6 shows a schematic of the experimental setup. A Ti:sapphire laser delivers tens of femtoseconds, nanojoule-level, $800 \mathrm{~nm}$ pulses at $80 \mathrm{MHz}$. Its output is stretched to $\sim 100 \mathrm{ps}$ in a double-pass stretcher. Subsequently, the pulses are amplified, reaching $50 \mathrm{~mJ}$ energy level at $10 \mathrm{~Hz}$ (Amplifier). The laser output ( $70 \mathrm{fs}, 25 \mathrm{~nm}$ bandwidth centered at $800 \mathrm{~nm}, \sim 10 \mathrm{~mJ}$, $\sim 13 \mathrm{~mm} 1 / e^{2}$ diameter), coming from the optical doublepass two-grating femtosecond compressor (Compressor), is split into two arms by a beam splitter (BS). The reflected beam is redirected, thanks to a simple reflective optic, onto a diffractive mask (MK) with a periodicity $d$ of $156 \mu \mathrm{m}$ and then focused onto a CCD by means of a $300 \mathrm{~mm}$ focal length achromatic doublet (L). Simultaneously, the transmitted beam is sent into an APE Compact LX-SPIDER ${ }^{[19]}$ for complete characterization in the spectral-temporal domain (phase retrieval and temporal measurement). Data from both instruments are collected by a computer, allowing a complete pulse characterization for each shot. The compressor parallelism was modified, moving the second grating (second and third passes) on the dispersion plane by a calibrated angle 


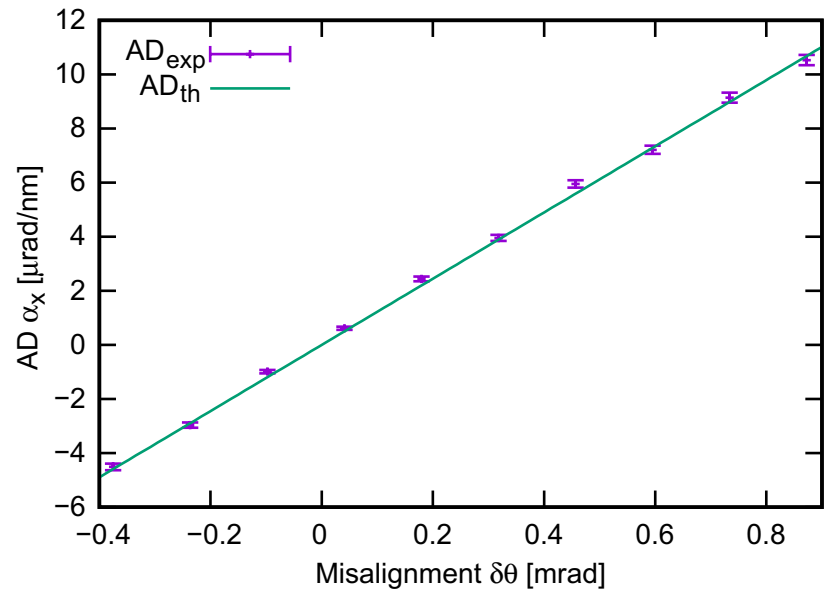

Figure 7. $\mathrm{AD}$ as a function of the introduced misalignment $\delta \theta$. Experimental results $\left(\mathrm{AD}_{\mathrm{exp}}\right)$ compared with the $4 \times 4$ Kostenbauder matrix formalism simulation $\left(\mathrm{AD}_{\mathrm{th}}\right)$ simulating a misaligned double-pass grating compressor.

$\delta \theta$ to introduce a controlled $\mathrm{AD}$, in the well-known $4 \times 4$ Kostenbauder formalism, given by

$$
\mathrm{PFT}=\frac{2 \lambda_{0} \tan \theta_{\mathrm{d}}}{c d \cos \theta_{\mathrm{i}}} \delta \theta,
$$

where $d$ is the groove distance, $c$ is the speed of light and $\theta_{\mathrm{i}, \mathrm{d}}$ are the incident and diffracted angle, respectively. It should be emphasized that the stretcher is not re-tuned at each induced $\delta \theta$ so the beam is not always maintained as short as $70 \mathrm{fs}$ but varies from 70 fs to 495 fs. Figure 7 shows the retrieved angular dispersion $\mathrm{AD}_{\text {exp }}$. The data were compared with the $4 \times 4$ Kostenbauder matrix model ${ }^{[20-22]}$, widely used to characterize $\mathrm{STCS}^{[2,23]}$, and showed excellent agreement. The average experimental error for the $\mathrm{AD}$ was $0.11 \mu \mathrm{rad} / \mathrm{nm}$, in agreement with the value estimated by the analysis algorithm.

In conclusion, $\mathrm{AD}$ retrieval is important for determining the performances of ultrashort high-power lasers. The proposed single-shot diagnostic, requiring a minimal number of optics and a compact layout, could be used as a realtime diagnostic, both on a high repetition rate (tens of hertz) laser system and on a low repetition rate, high-power, large laser system. Moreover, the ability to be applied also to stretched pulses will allow monitoring of $\mathrm{AD}$ across the entire amplification chain. The performances of the instrument were tested numerically and experimentally, proving to be robust and accurate over a wide range of parameters. Finally, thanks to the retrieval algorithm flexibility, more advanced analysis could be developed and implemented in future to perform real-time measurements of higher-order chromatic aberrations.

\section{References}

1. S. Akturk, X. Gu, P. Bowlan, and R. Trebino, J. Opt. 12, 093001 (2010).

2. S. Akturk, X. Gu, E. Zeek, and R. Trebino, Opt. Express 12, 4399 (2004).

3. M. Thévenet, D. E. Mittelberger, K. Nakamura, R. Lehe, C. B. Schroeder, J.-L. Vay, E. Esarey, and W. P. Leemans, Phys. Rev. Accel. Beams 22, 071301 (2019).

4. K. Osvay, A. P. Kovacs, Z. Heiner, G. Kurdi, J. Klebniczki, and M. Csatari, IEEE J. Sel. Top. Quantum Electron. 10, 213 (2004).

5. S. W. Jolly, O. Gobert, and F. Quéré, J. Opt. 22, 103501 (2020).

6. A. K. Sharma, R. K. Patidar, M. Raghuramaiah, P. A. Naik, and P. D. Gupta, Opt. Express 14, 13131 (2006).

7. Z. Li, N. Miyanaga, and J. Kawanaka, Opt. Lett. 43, 3156 (2018).

8. L. Gallmann, G. Steinmeyer, D. H. Sutter, T. Rupp, C. Iaconis, I. A. Walmsley, and U. Keller, Opt. Lett. 26, 96 (2001).

9. G. Figueira, L. Braga, S. Ahmed, A. Boyle, M. Galimberti, M. Galletti, and P. Oliveira, J. Opt. Soc. Am. B 36, 366 (2019).

10. S.-W. Bahk, C. Dorrer, and J. Bromage, Opt. Express 26, 8767 (2018).

11. Z. Li and J. Kawanaka, J. Opt. Soc. Am. B 37, 2595 (2020).

12. Z. Li and J. Kawanaka, Opt. Express 27, 25172 (2019).

13. Z. Li, J. Ogino, S. Tokita, and J. Kawanaka, Opt. Express 27, 13292 (2019)

14. M. Jean-Baptiste, C. Zhao, L.-M. Rodrigo, and O. Thomas, Opt. Lett. 45, 2207 (2020).

15. S. Akturk, M. Kimmel, P. O'Shea, and R. Trebino, Opt. Express 11, 491 (2003).

16. P. Yalamanchili, U. Arshad, Z. Mohammed, P. Garigipati, P. Entschev, B. Kloppenborg, J. Malcolm, and J. Melonakos, ArrayFire - A High Performance Software Library for Parallel Computing with an Easy-to-Use API (AccelerEyes, Atlanta, GA, 2015).

17. F. G. Bisesto, M. P. Anania, M. Bellaveglia, E. Chiadroni, A. Cianchi, G. Costa, A. Curcio, D. Di Giovenale, G. Di Pirro, M. Ferrario, F. Filippia, A. Galloa, A. Marocchinoa, R. Pompilia, A. Ziglerad, and C. Vaccarezzaa, Nucl. Instrum. Methods Phys. Res. A 909, 452 (2018).

18. M. Galletti, C. Coyle, P. Oliveira, M. Galimberti, F. G. Bisesto, and D. Giulietti, J. Inst. 14, C02005 (2019).

19. APE Compact LX SPIDER, https://www.ape-berlin.de/en/ spider-spectral-phase-interferometry-for-direct-electric-fieldreconstruction/compact-lx-spider/.

20. A. G. Kostenbauder, IEEE J. Quantum Electron. 26, 1148 (1990).

21. O. E. Martinez, IEEE J. Quantum Electron. 24, 2530 (1988).

22. V. Chauhan, J. Cohen, and R. Trebino, in Numerical Simulation of Optoelectronic Devices (2010), p. 115.

23. S. Akturk, X. Gu, P. Gabolde, and R. Trebino, Opt. Express 13, 8642 (2005). 